\title{
Further Notes on the Stieltjes Integral.
}

\author{
By J. HysLop.
}

Note 1.-On Young's Condition.

(Received 3rd April 1929. Read 8th June 1929.)

\section{§1. Preliminary Definition.}

The definition here used of the Stieltjes Integral is the same as that of a previous note, ${ }^{1}$ viz.:-

Let $f(x), \phi(x)$ be two real functions defined in $(a, b)$ a finite interval on the axis of the real variable $x$. Let $\Delta_{1}, \Delta_{2}, \ldots, \Delta_{n}$, be a finite set of sub-intervals which together make up $(a, b) . \Delta_{r} \phi$ denotes the increment of $\phi(x)$ in $\Delta_{r}$. Let $\xi_{r}$ be any point of $\Delta_{r}$, and form the sum

$$
\Sigma=\sum_{r=1}^{r=n} f\left(\xi_{r}\right) \Delta_{r} \phi
$$

Suppose that, given $\epsilon$, we can assign an $\eta$, such that every sum like $\Sigma$ differs from a fixed constant $I$ by less than $\epsilon$, provided only that, for all values of $r$ concerned, $\Delta_{r}<\eta$. Then $I$ is defined to be the value of the Stieltjes Integral $\int_{a}^{b} f(x) d \phi(x)$.

For further information on the Stieltjes Integral, including bibliography, the reader is referred to:

Hobson, Functions of a Real Variable, Vol. I (1927), 538-561, 662-668. Pollard, Quarterly Jo., 49 (1923), 73.

Hildebrandt, Bull. Amer. Math. Soc., 24 (1918), 177.

\section{§2. Introductory.}

In an important paper ${ }^{2}$ W. H. Young gave the following criterion for the existence of the Stieltjes Integral, which is now known by his name:

- 1 Proc. Edin. Math. Soc., 44 (1926), 79.

2 Proc. Lond. Math. Soc., 13 (1913), 113. 
If $\phi(x)$ is of bounded variation, the necessary and sufficient condition for the existence of the integral $\int_{a}^{b} f(x) d \phi(x)$ is that the variation of $\phi(x)$ over the set of points at which $f(x)$ is discontinuous be zero.

This theorem was proved by Young for the case in which $\phi(x)$ is monotone non-decreasing-the case in which he was primarily interested. He also gave a definition of the integral with respect to a function $\phi(x)$ of bounded variation, namely:

If $P(x), N(x)$ are the positive and negative variations of $\phi(x)$ in $(a, x)$,

$$
\int_{a}^{b} f(x) d \phi(x)=\int_{a}^{b} f(x) d P(x)-\int_{a}^{b} f(x) d N(x), \ldots \ldots \ldots
$$

provided the right side of the equality has a meaning.

With this definition it is clear that Young's theorem holds when $\phi(x)$ is of bounded variation. But several authors have assumed that equation (2) is true whenever the left side of the equation has a meaning in accordance with the definition of $\S 1$. This point has been noted by Miss R. C. Young, who has proved the validity of Young's criterion ${ }^{1}$ in the case of the $n$-dimensional Stieltjes Integral, when the function $\phi(P)$, with respect to which integration is carried out, is of bounded variation, and has in addition certain properties of continuity.

The question had, however, engaged the attention of the author about two years before the publication of Miss Young's results; in fact most of the present note contains work done then, which he was prevented by ill-health from publishing.

In the present note, a condition is used which is equivalent to Young's condition, but which does not assume any knowledge of the theory of Sets of Points. ${ }^{2}$ It is proved that the condition is necessary in all cases for the existence of the Stieltjes Integral, and that it is sufficient if $\phi(x)$ is of bounded variation.

1 Math. Zeitschr., 29 (1928), 217. See also additional note at the end of the present paper.

2 It seems to me that the Theory of the Riemann and Stieltjes Integrals is essentially concerned with intervals rather than with sets, and that it is of interest to develop it so far as possible without recourse to the theory of sets. 


\section{§3. General Conditions.}

We shall assume, to begin with, that

$$
m \leqslant f(x) \leqslant M \quad \text { for } a \leqslant x \leqslant b .
$$

Later, in $\S 6$, we shall be able to dispense with this condition.

In (1) we take any fixed set of subintervals of $(a, b)$, and consider the different values of $\Sigma$ obtained by varying $\xi_{r}$ in $\Delta_{r}$. Let $F_{r} . f_{r}$ be the upper and lower bounds respectively of $f(x)$ in $\Delta_{r}$. The upper bound $S$ of all sums like $\Sigma$ is then obtained by replacing $f\left(\xi_{r}\right)$ by $F_{r}$ when $\Delta_{r} \phi \geqslant 0$, and by $f_{r}$ when $\Delta_{r} \phi<0$.

The lower bound $s$ of the sums is similarly formed.

Then $S \geqslant \Sigma \geqslant s$.

Suppose now $\int_{a}^{b} f(x) d \phi(x)$ exists, and has the value $I$; then by definition, $|I-S|,|I-s| \leqslant \epsilon$ if $\Delta_{r}<\eta$

whence $S-s \equiv \sum_{r=1}^{r=n}\left(F_{r}-f_{r}\right)\left|\Delta_{r} \phi\right| \leqslant 2 \epsilon$ if $\Delta_{r}<\eta$

We have thus shown that:

Theorem 1. A necessary condition for the existence of $\int_{a}^{b} f(x) d \phi(x)$ is that, given $\delta$, we can assign $\eta$, such that, for every mode of subdividing $(a, b)$ into a finite set of subintervals $\Delta_{r}$. of length not exceeding $\eta$,

$$
\Sigma\left(F_{r}-f_{r}\right)\left|\Delta_{r} \phi\right|<. \delta . \quad \ldots
$$

A consequence is:

Theorem 2. If the condition of the last theorem is satisfied, and in particular if $\int_{a}^{b} f(x) d \phi(x)$ exists, the functions $f(x), \phi(x)$ have no common points of discontinuity.

In fact the condition of Theorem 1 is equivalent to Young's condition, which clearly includes Theorem 2. A separate proof of Theorem 2 may be given as follows:

Let $y$ be a point of discontinuity of $f(x)$, the saltus ${ }^{1}$ of $f(x)$ at $y$ being $2 k$; then for all intervals $\Delta$ containing $y$ as an interior point $F-f \geqslant 2 k$.

and so by hypothesis, $|\Delta \phi|<\frac{\delta}{2 k}$ if $\Delta<\eta$. Since $\delta$ is arbitrarily

I Hobson: loc. cit., pp. 295-303, in particular p. 301. 
small, all four limiting values of $\phi(x)$ at $y$ are equal. Again if $y$ be made a point of subdivision of $(a, b)$, then for all pairs of subintervals $\Delta_{1}, \Delta_{2}$ with $y$ as common end point, either $F_{1}-f_{1} \geqslant k$ or $F_{2}-f_{2} \geqslant k$; and hence, if $\Delta_{1}, \Delta_{2}$ be sufficiently small, either

$$
\left|\Delta_{1} \phi\right|<\frac{\delta}{k} \text { or }\left|\Delta_{2} \phi\right|<\frac{\delta}{k} \text {. }
$$

Thus $\phi(y)$ is equal either to the limiting values of $\phi(x)$ on the right at $y$ or to those on the left.

Thus $\phi(x)$ is continuous at $y$.

\section{\$4. Functions of Bounded Variation.}

Theorem 3. If $\phi(x)$ is monotone, the condition of Theorem 1 is sufficient for the existence of the Stieltjes Integral.

This Theorem is now classical, and need not be proved here. ${ }^{1}$

Theorem 4. If $\phi(x)$ is of bounded variation, and $\Phi(x)$ is the total variation of $\phi(x)$ in $(a, x)$, then, in order that $\int_{a}^{b} f(x) d \phi(x)$ exist, it is necessary and sufficient that $\int_{a}^{b} f(x) d \Phi(x)$ exist.

Suppose that $\phi(x)$ has external saltus ${ }^{2}$ only at the points $y_{1}, y_{2}, \ldots, y_{k}, \ldots$ of $(a, b)$. We assume that these points are infinite in number; the reasoning is simplified if this is not so. They are necessarily enumerable: ${ }^{3}$ being included in the discontinuities of $\phi(x)$. The external saltus $\sigma(y)$ at $y$ is defined to be the smaller of the two quantities $|\phi(y+0)-\phi(y)|,|\phi(y-0)-\phi(y)|$. The series $\sum_{k=1}^{\infty} \sigma\left(y_{k}\right)$ converges to a sum not exceeding $\frac{1}{2} \Phi(b)$. Thus, given $\theta$, we can choose $K$ so great that $\sum_{K+1}^{\infty} \sigma\left(y_{k}\right)<\theta$.

Suppose then that $\int_{a}^{l} f^{\prime}(x) d \phi(x)$ exists, and let $(a, b)$ be divided in any way into subintervals, each of length less than $\eta$, where $\eta$ is chosen to satisfy condition (4a).

${ }^{1}$ Cf. W. H. Youxa, loc. cit., pp. 133-134.

2 Hobsox: loc. cit., p. 301, 334.

3 Ibid., p. 325. 
From Theorem 2 we deduce that $\eta$ may be taken so small, that, in addition to restrictions already laid on it, $F_{y}-f_{r}<\delta / \Phi(b)$, in each of the subintervals, at most $2 K$ in number, which contain $y_{1}, y_{2}, \ldots, y_{k}$ as interior or end points.

Let $\Sigma^{(1)}$ denote summation over these subintervals, and $\Sigma^{(2)}$ summation over the remaining intervals of $(a, b)$.

Then $\Sigma^{(1)}\left(F_{r}-f_{r}\right) \Delta_{r} \Phi<\delta$.

Now we may suppose $\eta$ chosen so small that ${ }^{1}$

$$
\Sigma^{(2)}\left[\Delta_{r} \Phi-\left|\Delta_{r} \phi\right|\right] \leqslant 2 \sum_{K+1}^{\infty} \sigma\left(y_{k}\right)+\theta<3 \theta .
$$

Then $\Sigma^{(2)}\left(F_{r}-f_{r}\right) \Delta_{r} \Phi<\Sigma^{(2)}\left(F_{r}-f_{r}\right)\left|\Delta_{r} \phi\right|+3 \theta(M-m)$

$<2 \delta$ if $\theta$ be chosen so that $3 \theta(M-m)<\delta$.

Here, as previously, $m, M$ denote the bounds of $f(x)$ in $(a, b)$.

Hence, summing over $(a, b)$ if $\Delta_{i}$. is sufficiently small,

$$
\Sigma\left(F_{r}-f_{i}\right) \Delta_{i} \Phi<3 \delta \text {. }
$$

Thus, by Theorem $3, \int_{a}^{b} f(x) d \Phi(x)$ exists.

This proves necessity of the condition.

Now let $P(x), N(x)$ be the positive and negative variations of $\phi(x)$ in $(a, x)$,

so that $2 P(x)=\Phi(x)+\phi(x)-\phi(a)$

$$
2 N(x)=\Phi(x)-\phi(x)+\phi(a) .
$$

Then $P(x), N(x)$ are monotone increasing functions, and

$$
\Delta_{r} P(x), \Delta_{r} N(x) \leqslant \Delta_{r} \Phi(x) .
$$

Hence, by Theorem 3 , if $\int_{a}^{b} f(x) d \Phi(x)$ exists, so do $\int_{a}^{b} f(x) d P(x)$, $\int_{a}^{b} f(x) d N(x)$; and clearly $\int_{a}^{b} f(x) d \phi(x)=\int_{a}^{b} f(x) d P(x)-\int_{a}^{b} f(x) d N(x)$.

Thus the condition is proved sufficient.

In the first part of the above proof, the only use made of the existence of $\int_{a}^{b} f(x) d \phi(x)$ is to ensure that the condition of Theorem 1 is satisfied.

The following may therefore be deduced as a corollary:

1 See additional note at the end of this paper. 
Theorem 5. If $\phi(x)$ is of bounded variation, the condition of Theorem 1 is sufficient as well as necessary.

\section{§5. A Deduction.}

Theorem 6. If $\phi(x)$ is of bounded variation, and $\int_{a}^{b} f(x) d \phi(x)$ exists, then $\int_{a}^{x}|f(x)| d \Phi(x)$ also exists, and is the total variation in $(a, x)$ of $\int_{a}^{x} f(x) d \phi(x) . \quad[a \leqslant x \leqslant b]$.

To prove the existence of $\int_{a}^{b}|f(x)| d \Phi(x)$ we use Theorem 3, noting that if $M_{r}, m_{r}$ are the upper and lower bounds of $|f(x)|$ in $\Delta_{r}$, then

$$
M_{r}-m_{r} \leqslant F_{r}-f_{i \cdot}
$$

The remaining part of the Theorem has already been dealt with in the author's previous note. ${ }^{1}$

§ 6. The Boundedness of $f(x)$.

In all the above working it has been assumed that $f(x)$ is bounded. It is however clear, that if $y$ is a point in the neighbourhood of which $f(x)$ is unbounded, and if $\int_{a}^{b} f(x) d \phi(x)$ exists, or any of our hypotheses is fulfilled, then there must exist an interval with $y$ as interior point, in which $\phi(x)$ is constant. Such an interval contributes nothing to the approximating sums, to the integral, or to the other sums used above. Hence the existence of the various integrals concerned, and the fulfilment of the conditions laid down are unaffected by the behaviour of $f(x)$ in any such interval. We may therefore dispense with this restriction in the statement of the theorems.

Note added 22nd April 1929. (See footnote p. 235.)

Miss Young's condition of continuity of $\phi(\Sigma)$ is unnecessarily stringent. Her proof of Theorem IV, p. 229 of her paper, remains valid if, for every mode of subdividing the range of integration into

1 Loc. cit., p. 84. 
elements $\Delta$ of sufficiently small diameter, $\Sigma|\Delta \phi|$ differs from the variation of $\phi(\Sigma)$ by an arbitrarily small amount. The class of functions $\phi(\Sigma)$ which satisfy this condition includes, and is wider than, the class of continuous functions of bounded variation. Thus in one dimension it includes all functions of bounded variation without external saltus.

Note added 26th April 1929, re Inequality (5), p. 238.

If $\phi(x)$ is a function of bounded variation in $(a, b)$ with external saltus $\sigma\left(y_{k}\right)$ at the points $y_{k}$, the lower limit, as Max. $\Delta_{r} \rightarrow 0$, of $\Sigma\left|\Delta_{r} \phi\right|$ is $\left.\operatorname{Var} . \phi(x)\right]_{a}^{b}-2 \Sigma \sigma\left(y_{k}\right)$. Thus in particular, given $\theta$, we can choose $\eta$ so that if $\Delta_{r}<\eta$

$$
\sum \Delta_{r} \phi>\operatorname{Var} . \phi(x)-2 \sum \sigma\left(y_{k}\right)-\theta .
$$

A proof of this theorem may readily be constructed on the lines of Hobson, p. 332-335. Professor Hobson's enunciation of the theorem [p. 335, §247] differs slightly from the above, but his proof requires but little alteration to justify the more stringent statement. 